\title{
The human frontal operculum is involved in visuomotor performance monitoring
}

Felix Quirmbach ${ }^{1,2}$ and Jakub Limanowski ${ }^{1,2}$

${ }^{1}$ Faculty of Psychology, Technische Universität Dresden, Dresden, Germany

${ }^{2}$ Centre for Tactile Internet with Human-in-the-Loop, Technische Universität Dresden, Dresden, Germany

Correspondence: Felix Quirmbach, Technische Universität Dresden, CeTI - Cluster of Excellence, 01062 Dresden, Germany, Phone: +49 351 46334941, Email: felix.quirmbach@tu-dresden.de 


\section{Abstract}

1 For adaptive goal-directed action, the brain needs to monitor action performance and detect errors.

2 The corresponding information may be conveyed via different sensory modalities; for instance, visual and proprioceptive body position cues may inform about current manual action performance.

Thereby, contextual factors such as the current task set may also determine the relative importance

5 of each sensory modality for action guidance. Here, we analysed human behavioral, $\mathrm{FMRI}$, and MEG

6 data from two VR-based hand-target phase matching studies to identify the neuronal correlates of

7 performance monitoring and error processing under instructed visual or proprioceptive task sets. Our

8 main result was a general, modality-independent response of the bilateral FO to poor phase matching

9 accuracy, as evident from increased BOLD signal and increased gamma power. Furthermore, functional

10 connectivity of the bilateral FO to the right PPC increased under a visual vs proprioceptive task set.

11 These findings suggest that the bilateral FO generally monitors manual action performance; and, moreover, that when visual action feedback is used to guide action, the FO may signal an increased need for control to visuomotor regions in the right PPC following errors.

Key words: Action; frontal operculum; sensorimotor integration; performance monitoring; visuoproprioceptive integration 
14

\section{Introduction}

To effectively perform goal-directed action in the environment, the brain needs to monitor motor performance and detect errors, so that it can enable adaptive changes in behavior (Diedrichsen, 2005; Klein et al., 2007; Suminski et al., 2007; Ullsperger et al., 2014). During performance monitoring, the predicted outcome of one's actions selected based on current goals (i.e., task set) is compared with actual sensory feedback, and behavioural changes are initiated if a mismatch between both is detected (Ullsperger et al., 2014). The neurofunctional basis of performance monitoring and error correction has been illuminated by recent brain imaging and electrophysiological work. Specifically, a 'salience network' comprising, among others, the dorsal anterior cingulate cortex, the bilateral insular cortex and the inferior frontal gyri, is assumed to integrate sensory input, responding to behaviourally salient stimuli-behavioural errors - with increased activation (Ham et al., 2013; Seeley et al., 2007; Sridharan et al., 2008; Uddin, 2021). Thereby regions like e.g. the frontal operculum (FO, also anterior insular cortex, IC(Billeke et al., 2020; Cieslik et al., 2015; Higo et al., 2011; Klein et al., 2013; Sridharan et al., 2008) may signal a need for increased cognitive control to the executive control network, consisting (among other regions) of the lateral prefrontal cortices, the posterior parietal cortex, presupplementary motor area and the inferior parietal lobule(Uddin, 2021; Ullsperger et al., 2010). This network, in turn, may direct attentional resources to the relevant stimuli, driving behavioural adaptions (Menon \& Uddin, 2010; Sridharan et al., 2008).

Notably, action performance and error may be conveyed via different sensory modalities; in manual action, for instance, via visual and proprioceptive cues about body position. In the context of body representation for action, visual and proprioceptive body position cues can be weighted depending on the current context; e.g., based on their relative relevance for the specific task at hand (Lebar et al., 2017; Sober \& Sabes, 2005; van Beers et al., 1999). Recently, we have used virtual reality (VR) to examine this contextual sensory weighting during action under conflicting visual (virtual) and proprioceptive (real, unseen) body position feedback. Our functional magnetic resonance imaging (fMRI) and magnetoencephalography (MEG) studies (Limanowski et al., 2020; Limanowski \& Friston, 
2020) specifically shed light on the effects of adopting a visual vs proprioceptive attentional set during goal-directed manual action tasks, demonstrating that participants' can prioritise either modality over the other; and we observed corresponding changes of neuronal gain in the respective sensory (visual and proprioceptive) brain regions. However, while the effects of adopting an attentional set on sensory processing could be seen clearly, these studies did not investigate the specific neural correlates of flexible performance monitoring in these settings.

Here, we aimed to close this gap. We therefore re-analysed the behavioural, fMRI, and MEG data from the above studies, correlating participants' task performance with hemodynamic and oscillatory activity. Based on the above literature, we expected task inaccuracy to be reflected by activity in the performance monitoring network and, potentially, also in fronto-parietal attentional areas. Our second research question was whether performance monitoring would be modality specific (i.e., involve different brain regions when vision vs proprioception was task relevant) or general. Therefore, we also tested for task set dependent differences in brain activity and connectivity. 


\section{Materials and Methods}

\section{Participants}

For this study, we reanalysed fMRI and MEG data acquired by (Limanowski et al., 2020; Limanowski \& Friston, 2020). Healthy, right-handed volunteers with normal or corrected-to-normal vision participated in both experiments after providing written informed consent. The fMRI study included 16 subjects ( 8 female, mean age 27 , range $21-37$ ), the MEG study in included 18 subjects ( 9 female, mean age 29, range 21-39). Both experiments were approved by the local research ethics committee (University College London) and conducted in accordance with these approvals.

\section{Experimental design and task}

Participants wore an MR compatible data glove (5DT Data Glove MRI, 1 sensor per finger, 8 bit flexure resolution per sensor, $60 \mathrm{~Hz}$ sampling rate, communication with the PC via USB) on their right hand. The glove measured each finger's flexion via sewn-in optical fibre cables, and was carefully calibrated to fit each participant's movement range prior to scanning. Recorded hand movement data was used to control a photorealistic virtual hand $(\mathrm{VH})$ model, moving in accordance to the participant's hand movements and presented as part of a virtual reality task environment. This virtual environment, consisting of the $\mathrm{VH}$, a fixation dot and task instructions, was created in the open-source 3D computer graphics software Blender ( $\underline{\mathrm{http}}$ ///blender.org). The environment was presented via a projector on a screen (for details see (Limanowski et al., 2020; Limanowski \& Friston, 2020).

Participants were instructed to perform repetitive right-hand grasping movements, paced by oscillatory $(0.5 \mathrm{~Hz})$ size changes $(12 \%)$ of the central fixation dot, resulting in a non-spatial phase matching task: Thus, participants had to match the fully open hand position with the biggest dot size and, conversely, the fully closed hand with minimal dot size. They performed the task in movement blocks of $32 \mathrm{~s}$ (16 close-and-open movements; the last movement was signaled by brief blinking of fixation dot), separated by $16 \mathrm{~s}$ rest periods during which only the fixation dot was visible. All participants trained extensively before scanning. Note that this task was, therefore, not designed to 
investigate visuomotor adaptation or learning, but maintaining hand-target phase matching during a sustained visual vs proprioceptive attentional task set.

Before the start of each movement block, participants were instructed to match the phase of the fixation dot with either the seen VH model or their unseen real hand. In half of the conditions, a lag was introduced to the virtual hand's movements; i.e., the virtual hand movements lagged behind the actually executed movements. In the fMRI experiment, a lag of $267 \mathrm{~ms}$ was introduced in the second half of each movement block; in the MEG experiment, a lag of 500 ms was presented as a separate block. Note that under incongruence, only one modality could be aligned with the target phase, which resulted in a misalignment of the other one. The task instruction ('VIRTUAL' or 'REAL') were presented $2.5 \mathrm{~s}$ before start of each movement block for $2 \mathrm{~s}$, and the color of the fixation dot reminded participants of the current condition thorough the block. In the fMRI experiment (Limanowski \& Friston, 2020), we had additionally varied the visibility (high or low) of the virtual hand during half of the movement blocks. However, we found no differences in performance between different visibility levels; and in our present reanalysis, there were no significant differences between visibility levels either. Therefore, we present the differential fMRI task contrasts in terms of VH vs RH task, summing over high and low visibility levels in each condition. In sum, despite minor technical differences between $\mathrm{fMRI}$ and MEG experiments, both can be described as a balanced $2 \times 2$ factorial design with the factors task (VH vs RH) and congruence (congruent vs incongruent).

\section{Behavioural Data Analysis}

In our previous analyses, we examined the neuronal correlates of the instructed task set; and only analysed condition specific differences in average performance (Limanowski et al., 2020; Limanowski \& Friston, 2020). In the present study, we examined the neuronal correlates of phase matching accuracy (i.e., fluctuations around those average performances).

To quantify hand-target phase matching (in)accuracy, we calculated the root mean square error (RMSE) of the difference between the target position (i.e., the position within the oscillatory cycle) and 
the position of the task relevant hand (i.e., the position within the grasping cycle, averaging the recorded finger position data per hand). Thus, the virtual hand position was evaluated for the $\mathrm{VH}$ condition movements and the real hand position for the $\mathrm{RH}$ condition. For construction of the fMRI/MEG regressors, we binned the resulting RSME values into $1 \mathrm{~s}$ time windows, each centred on a time point of minimum or maximum target size, corresponding to the hand fully closed or opened if moved synchronously with the target. To focus on within-subject fluctuations in performance, rather than between-subject differences, the overall RSME across the entire experiment was normalized for each single subject (i.e. minimum and maximum performance error value was equal across participants; 0 and 1, respectively). The resulting RSME values were assigned to one regressor per experimental condition ( $\mathrm{VH}$ congruent, $\mathrm{VH}$ incongruent, $\mathrm{RH}$ congruent, $\mathrm{RH}$ incongruent), and demeaned separately to reflect only variation around the condition mean. To evaluate if the variance of the phase matching differed between the $\mathrm{VH}$ and $\mathrm{RH}$ conditions, we calculated a paired t-test on the participants' variance of phase matching RMSE within each condition.

The amplitude of the hand movement at each time point was calculated via a cubic spine interpolation of the respective minimum and maximum hand position values in each time window. The resulting time series was de-meaned per condition as well, and used as noise regressor for the following fMRI and MEG analysis (see below). Additionally, we tested for correlations between performance error and movement amplitude by calculating the Pearson correlation coefficient of both regressors for each participant; and testing it for significance (i.e., significant difference from zero) with a t-test on the group level. Similarly, we calculated the correlation between performance error and fMRI head movements (realignment parameters), via subject-level Pearson correlation and group-level t-test, adjusted for multiple comparisons for the six realignment parameters. All analyses were performed using MATLAB (MathWorks, Natick, MA, United States). 
FMRI Data Preprocessing and Analysis

127

128

All analyses were performed using MATLAB (MathWorks, Natick, MA, United States) and SPM12.6 (Wellcome Trust Centre for Neuroimaging, University College London, https://www.fil.ion.ucl.ac.uk/spm/).

We reused the preprocessed fMRI data by (Limanowski \& Friston, 2020). The fMRI data had been acquired using a $3 T$ scanner (Magnetom TIM Trio, Siemens), equipped with a 64-channel head coil. $\mathrm{T}_{2}{ }^{*}$-weighted images were acquired using gradient echo-planar imaging sequence (voxel size $=3 \times 3 \times 3 \mathrm{~mm}^{3}$, matrix size $=64 \times 72, \mathrm{TR}=3.36 \mathrm{~s}, \mathrm{TE}=30 \mathrm{~ms}$, flip angle $=90^{\circ}$ ).

We fitted a general linear model (GLM, $128 \mathrm{~s}$ high-pass filter) to each participant. Each condition (VH, $\mathrm{RH}$ ) was modelled with a boxcar function as a $32 \mathrm{~s}$ movement block; we added a parametric modulator $(1 /-1)$ to each condition encoding the first half of each block as congruent $(-1)$ and the second half as incongruent (1) movement periods. Additionally, we included a regressor encoding the (de-meaned) RSME values for each conditions; the values were re-sampled to match the $3.36 \mathrm{~s}$ scan length prior to this. Regressors modelling the task instructions and movement amplitude were added to the GLM alongside the realignment parameters as regressors of no interest.

For each subject, we calculated contrast images of each RSME regressor against the baseline. These were then entered into a group-level flexible factorial design, with the factors task ( $\mathrm{VH}$ or $\mathrm{RH}$ ) and congruence (congruent, incongruent), and an additional factor modelling the subject constants. To assess potential differences between congruent and incongruent movement periods, we calculated separate first-level GLMs, in which the RSME values of the second movement half were inverted; this effectively encoded the contrast congruent-incongruent. The resulting contrast images were entered into an analogous group-level GLM as described above.

Group-level results were assessed for statistical significance using a voxel-wise threshold of $p<0.05$, family-wise error ( $\left.p_{\mathrm{FWE}}\right)$ corrected for multiple comparisons. We projected the resulting statistical maps onto the mean normalized structural image or rendered it on SPM12's brain template. The 
unthresholded T-maps corresponding to the contrasts reported here can be inspected online at https://neurovault.org/collections/GGWQTGSI. For anatomical reference we used the SPM Anatomy toolbox (Eickhoff et al., 2005).

\section{MEG Data Preprocessing and Analysis}

MEG signals had been acquired using a 275-channel whole-head setup with third-order gradiometers (CTF Omega, CTF MEG International Services LP, Coquitlam, Canada) at a sampling rate of $600 \mathrm{~Hz}$. Following the original analysis by (Limanowski et al., 2020), the MEG data were high-pass filtered ( $1 \mathrm{~Hz}$ ), downsampled to $300 \mathrm{~Hz}$, and epoched into trials of $2 \mathrm{~s}$ each (each corresponding to a full target oscillation/grasping cycle).

In the main (sensor space) MEG data analysis, we looked for spectral power differences under 'steadystate' assumptions; i.e., treating the spectral profile as a 'snapshot' of performance dependent responses as manifest in quasi-stationary power spectra (Donner \& Siegel, 2011; Friston et al., 2019; Moran et al., 2008). We computed trial-by-trial power spectra in the $0-98 \mathrm{~Hz}$ range using a multi-taper spectral decomposition (Thomson, 1982) with a spectral resolution of $+-2 \mathrm{~Hz}$. The spectra were logtransformed, converted to volumetric scalp $\mathrm{x}$ frequency images-one image per trial-with two spatial and one frequency dimension (Kilner \& Friston, 2010), and smoothed with a Gaussian kernel with full width at half maximum of $8 \mathrm{~mm} \times 8 \mathrm{~mm} \times 4 \mathrm{~Hz}$. The resulting images were entered into a general linear model (GLM) using a within-subject ANOVA with the respective RSME values as a covariate (first-level analysis). As in the fMRI analysis, movement amplitude was moreover included as a covariate of no interest to capture movement related fluctuations. Contrast images were then calculated for each condition's accuracy covariate. These contrast images were then entered into a group-level GLM using a flexible factorial design including the two within-subject experimental factors (task and congruence), and a factor modelling the between subject variance. The statistical parametric maps obtained from the respective group-level contrasts were evaluated for significant effects using a threshold of $p<0.05$, family-wise error ( $p_{\mathrm{FWE}}$ ) corrected for multiple comparisons at the peak (voxel) level. 
As a post-hoc analysis, source localization of trial-by-trial correlation of gamma band power with performance error was performed using a variational Bayesian approach with multiple sparse priors (Litvak \& Friston, 2008). Source localization was performed in the $34-88 \mathrm{~Hz}$ range (which was the range of effects in the spectral analysis thresholded at $p<0.001$, uncorrected). As we had already performed an analogous localization on the fMRI data (see above), we could use the superior spatial acuity of fMRI to improve MEG source localization; i.e., the $\mathrm{fMRI}$ activations (thresholded at $p<0.001$, uncorrected) were used as empirical (spatial) priors for the Bayesian inversion routine (Henson et al., beamforming approach (Belardinelli et al., 2012). This produced very similar results; i.e., the strongest effects were localized to the bilateral inferior frontal gyri, including the FO (a further, weaker source 3D images and entered into a group-level t-test. Since the significance of the effects on spectral responses had already been established with the sensor space analysis, the ensuing statistical parametric maps were displayed at a threshold of $p<0.05$, uncorrected, rendered on SPM's smoothed average brain template. The unthresholded T-map corresponding to the source localization can be inspected online at https://neurovault.org/collections/GGWQTGSI.

\section{FMRI functional connectivity analysis}

In our main analysis (see above), we identified brain areas that showed a significance response to phase matching inaccuracy. The fMRI and MEG results consistently highlighted the bilateral FO (while further $\mathrm{FMRI}$ activations were found in the SMA and the dIPFC). the dIPFC. However, following the clear response of the FO to poor task performance in general, we

199 now asked whether these areas would change their connectivity to other (potentially, task relevant) brain areas depending on whether the inaccuracy was registered during the $\mathrm{VH}$ or $\mathrm{RH}$ task; i.e., while participants focused either on visual $(\mathrm{VH})$ or proprioceptive $(\mathrm{RH})$ action feedback. 
To answer this question, we used psychophysiological interaction (PPI) analysis for fMRI data. This analysis aims to explain neuronal activity other brain areas in terms of an interaction between psychological factors (the specific task condition) and physiological factors (the BOLD signal time course in the region of interest (Friston et al., 1997; O'Reilly et al., 2012). The resulting interaction (PPI) reveals voxels in the brain increase their connectivity with a specific seed region in a given context; e.g., in a specific task condition. Note that task dependent changes in connectivity per se (i.e., between limited the connectivity analysis to the fMRI data.

For the PPI analysis, we calculated separate GLMs with concatenated runs for each participant, and thus identified subject-specific peaks of the main effects observed on the group level. The individual peaks were defined as the maximum effect within a $10 \mathrm{~mm}$ radius sphere of the respective group-level maximum (see Table 1). From these individual peaks, we extracted the BOLD signal of the seed regions, as the first eigenvariate of activity across all voxels in a $4 \mathrm{~mm}$ radius sphere centred on the participantspecific peak. For three subjects where no effect could be identified for the specific SMA seed region as well as one case were no effect was found for the dIPFC region, we resorted to the group level maximum for seed region localization.

The SPM12.6 PPI routine was then used to form the interaction between the psychological factor and the seed region's summarized BOLD signal time course. Note that while the seed regions were identified per their significant response to phase matching inaccuracy (Fig. 2), our psychological factor was the task set; i.e., the instructed hand modality at the beginning of each movement block (VH vs 
bioRxiv preprint doi: $\mathrm{https}$ //doi.org/10.1101/2021.10.21.465314; this version posted October 22, 2021. The copyright holder for this preprint (which was not certified by peer review) is the author/funder, who has granted bioRxiv a license to display the preprint in perpetuity. It is made available under aCC-BY-NC-ND 4.0 International license.

term, a second GLM was constructed for each participant, including the interaction, the seed region's

extracted signal, the task set and the realignment parameters as regressors of no interest.
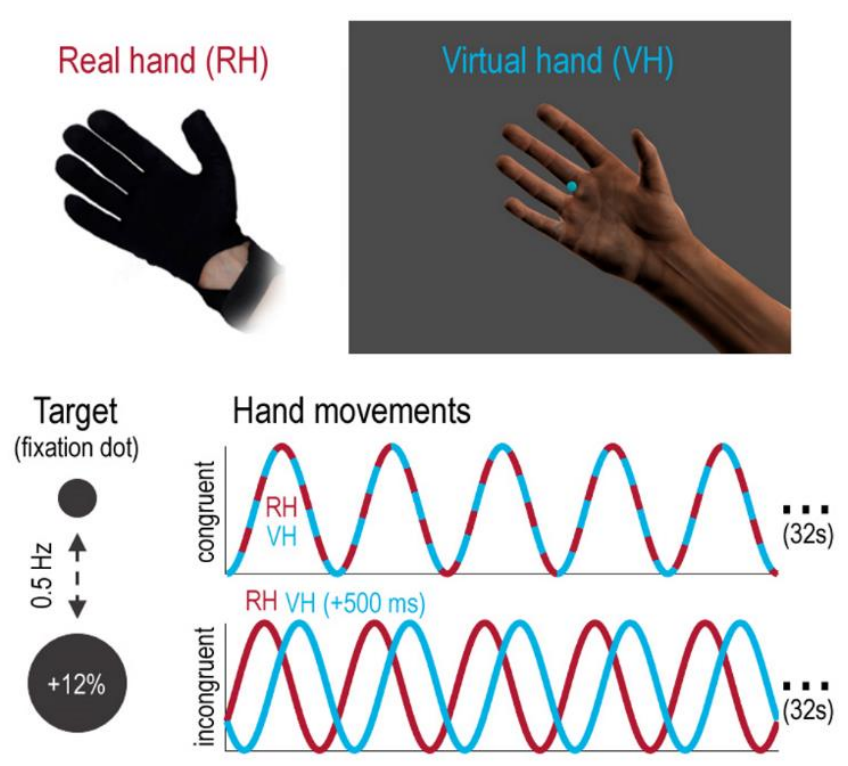

Figure 1. Phase matching task. Participants controlled a photorealistic virtual hand model (VH) with a data glove worn on their real hand ( $\mathrm{RH})$; the $\mathrm{RH}$ was occluded from view, while participants saw the VH at all times. Participants had to match the oscillatory phase of a virtual target (fixation dot, changing its size sinusoidally at $0.5 \mathrm{~Hz}$ ) with right hand grasping movements (i.e., open at maximum target size, closed at minimum size). Thereby, participants were instructed to match the target's oscillatory phase with the grasping movements of either the $\mathrm{VH}$ or the unseen $\mathrm{RH}$. These instructions were intended to induce a specific task set, in which either visual or proprioceptive movement information was task relevant. In half of all trials, $\mathrm{RH}$ and $\mathrm{VH}$ moved congruently ('congruent'), while in the other half of the trials ('incongruent'), the movements of the $\mathrm{VH}$ were delayed (e.g., by $500 \mathrm{~ms}$ ) with respect to the actually executed movements (RH); this introduced visuoproprioceptive incongruence. Reprinted from Limanowski et al. (2020). 


\section{Results}

235

236

237

238

239

240

241

242

243

\section{Behavioural results}

In both studies, participants were able to follow the task instructions; i.e., to keep the instructed modality's (vision or proprioception) grasping movements aligned with the phase of the dot (see the original studies, Limanowski et al., 2020; Limanowski \& Friston, 2020, for details and condition specific differences between task performance). In the MEG data, phase matching was on average significantly more variable in the $\mathrm{RH}$ compared to the $\mathrm{VH}$ conditions $\left(t_{(17)}=2.27, \mathrm{p}<0.05\right)$; but this was not the case in the fMRI data $\left(t_{(15)}=0.55\right.$, n.s. $)$. On average, phase matching accuracy correlated weakly but significantly with movement amplitude (mean Pearson's $r=.27, t_{(15)}=6.69, p<0.001$ for fMRI; mean $r$ $=.18, t_{(17)}=5.08, p<0.001$ for MEG) but not significantly with the fMRI realignment parameters (all $|r|<0.02$, n.s. $)$.

\section{FMRI results}

In our main fMRI analysis, we sought to identify brain regions in which neuronal activity correlated with phase matching (in)accuracy (Fig. 2). A significant ( $p_{\mathrm{FWE}}<0.05$ ) main effect of inaccuracy was observed in the bilateral FO, the left SMA, and the left dIPFC (see Table 1 and Fig. 2). More liberal thresholds ( $p<0.001$, uncorrected) revealed further activation clusters in the right middle and superior frontal gyri, the precuneus, the medial cingulate cortex (MCC), the right middle temporal gyrus (MTG), and bilaterally in the cerebellum (cf. the render in Fig. 2). Conversely, a significant main effect of accuracy was found in the left pre- and postcentral gyrus, corresponding to the primary motor cortex (M1). No other comparisons (i.e., contrasting the effects of accuracy between task conditions, delay, or visual salience levels; see Methods) yielded significant effects. At uncorrected thresholds $(p<0.001)$, voxels in several brain areas showed a stronger correlation with task inaccuracy under the VH task than under the RH task; namely, in the MCC, the bilateral FO, the right MTG, the left cerebellum, the right dIPFC, and the bilateral posterior parietal cortex (PPC, peak within the intraparietal sulcus, IPS). 

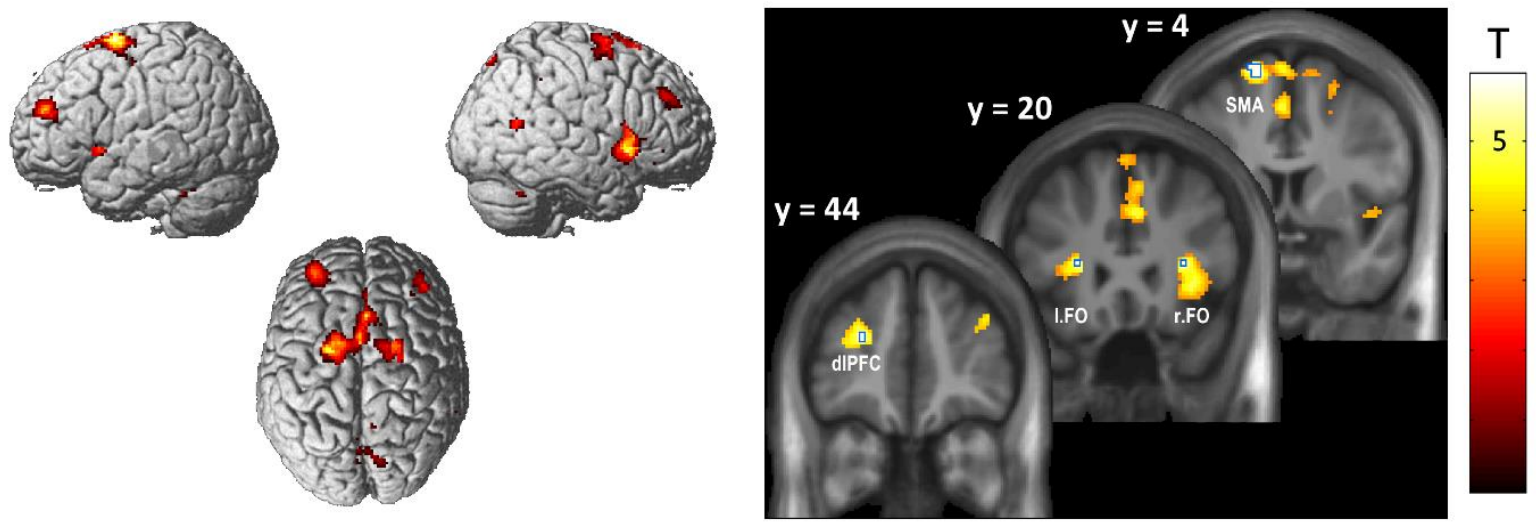

Figure 2. BOLD signal increases related to phase matching inaccuracy. The renders (left) and slice overlays (right) show brain areas in which hemodynamic activity was correlated with the relative inaccuracy of hand-target phase matching (displayed at $p<0.001$, uncorrected). Significant activations ( $p_{\mathrm{FWE}}<0.05$; voxels outlined in blue on the slice overlays) were located in the bilateral FO, the left SMA, and the left dIPFC.

Table 1. Significant $\left(p_{\mathrm{FWE}}<0.05\right)$ activations for all reported fMRI contrasts.

\begin{tabular}{|c|c|c|c|c|c|c|}
\hline Anatomical location & Voxels & \multicolumn{3}{|c|}{ MNI $(x, y, z)$} & Peak T & Peak $p_{\mathrm{FWE}}$ \\
\hline \multicolumn{7}{|c|}{ Correlation with phase matching inaccuracy } \\
\hline L. Insula (FO) & 1 & -26 & 20 & 12 & 5.92 & 0.012 \\
\hline L. Superior frontal gyrus (SMA) & 13 & -16 & 4 & 70 & 5.90 & 0.014 \\
\hline $\begin{array}{l}\text { L. Middle frontal gyrus / frontal pole } \\
\text { (dIPFC) }\end{array}$ & 2 & -26 & 44 & 24 & 5.80 & 0.018 \\
\hline R. Insula (FO) & 3 & 30 & 22 & 12 & 5.72 & 0.023 \\
\hline \multicolumn{7}{|c|}{ Correlation with phase matching accuracy } \\
\hline \multirow{3}{*}{ L. Pre- and postcentral gyrus (M1) } & 6 & -32 & -18 & 38 & 6.18 & 0.006 \\
\hline & 3 & -28 & -22 & 66 & 5.78 & 0.019 \\
\hline & 1 & -24 & -24 & 62 & 5.49 & 0.044 \\
\hline
\end{tabular}

\section{MEG results}

The MEG sensor space analysis revealed that phase matching inaccuracy was associated with significantly increased spectral power in the gamma frequency range over mid-frontal sensors (main effect; peak at $52 \mathrm{~Hz}, \mathrm{~T}=5.31, p_{\mathrm{FWE}}<0.05$; see Fig. $3 \mathrm{~A}$ ). These spectral effects were source-localized to the bilateral inferior frontal gyri, including the bilateral FO (Fig. 3B). No other spectral power 


\section{A}
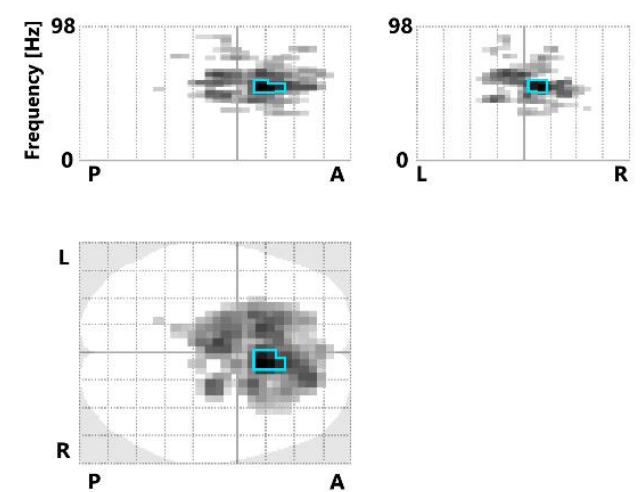

B

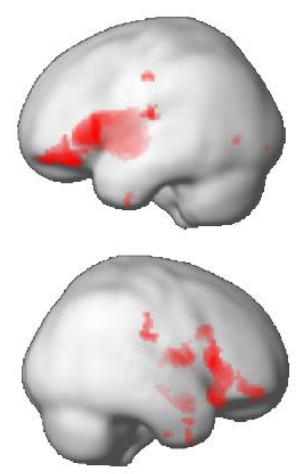



Figure 3. Spectral power increases related to phase matching inaccuracy. A: The 'glass brain' (maximum intensity) projections show the sensor level scalp-frequency maps of spectral power correlated with the relative inaccuracy of hand-target phase matching (the darkest voxels show the strongest effect along the respective projection; the maps are thresholded at $p<0.001$, effects significant at $p_{\mathrm{FWE}}<0.05$ are outlined in blue; the top plots have one frequency dimension, $0-98 \mathrm{~Hz}$, and one spatial dimension, $\mathrm{P}-\mathrm{A}=$ posterioranterior, $\mathrm{L}-\mathrm{R}$ = left-right; the bottom plot has two spatial dimensions). B: Renders (left) and slice overlay (right) showing the corresponding source localization of the correlation to regions around the FO.

\section{Functional connectivity analysis}

The above $\mathrm{fMRI}$ activations and source-localized MEG gamma power consistently suggested that periods of poor phase matching activated the bilateral FO, in line with previous literature that had established this region's role in error processing and performance monitoring (see Introduction). However, we did not find any significant difference between conditions (i.e., between visual and proprioceptive task sets). Therefore, we next performed a connectivity (PPI) analysis on the fMRI data to explore whether task relevant brain areas would change their connectivity to the FO depending on the instructed task condition ( $\mathrm{VH}$ or $\mathrm{RH}$ ).

This analysis revealed a significantly increased coupling of several brain areas with the bilateral FO during the $\mathrm{VH}$ task > RH task, most strongly expressed in the right inferior parietal lobe (IPL, see Fig. 4A and Table 2). The increase in coupling with the right IPL was evident for both the left and right FO independently, as revealed by an additional 'null' conjunction analysis (Fig. 4B; i.e., a conjunction of 
bioRxiv preprint doi: https//doi.org/10.1101/2021.10.21.465314 this version posted October 22, 2021. The copyright holder for this preprint (which was not certified by peer review) is the author/funder, who has granted bioRxiv a license to display the preprint in perpetuity. It is made available under aCC-BY-NC-ND 4.0 International license.

voxels activated in the PPI with the left FO and PPI with the right FO, each thresholded at $p<0.001$,

uncorrected). Correspondingly, there were no significant differences in coupling between the left and

right FO. A supplementary analysis testing for potential coupling differences with the FO during $\mathrm{VH}$

A $\quad$ L $+R . F O \times(V H>R H)$
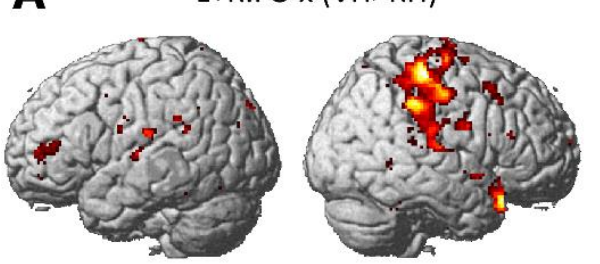

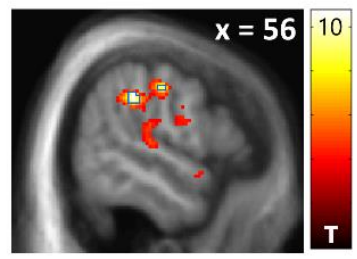

B L.FO $x(\mathrm{VH}>\mathrm{RH}) \cap$ R.FO $x(\mathrm{VH}>\mathrm{RH})$

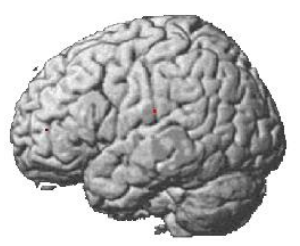



Figure 4. Task-dependent connectivity changes of the bilateral FO. A: Brain areas showing increased coupling with the bilateral FO during the $\mathrm{VH}$ task relative to the $\mathrm{RH}$ task (displayed at $p<0.001$, uncorrected). The strongest effects were located in the right IPL (voxels significant at $p_{\mathrm{FWE}}<0.05$ are outlined in blue). B: A corresponding 'null' conjunction contrasts confirmed this increased task dependent coupling with the right IPL for the left and right FO independently (each PPI contrast thresholded at $p<0.001$, uncorrected).

Table 2. Brain areas showing significant $\left(p_{\mathrm{FWE}}<0.05\right)$ coupling increases with the bilateral FO during the $\mathrm{VH}$ task $>$ RH task.

\begin{tabular}{|l|c|c|c|c|c|c|}
\hline \multicolumn{1}{|c|}{ Anatomical location } & Voxels & \multicolumn{3}{|c|}{ MNI (x, y, z) } & Peak T & Peak pFWE \\
\hline $\begin{array}{l}\text { R. Inferior parietal lobe / } \\
\text { supramarginal gyrus (IPL/SMG) }\end{array}$ & 15 & 56 & -32 & 38 & 10.71 & 0.002 \\
\hline R. Postcentral gyrus (S1) & & & & & & \\
\hline R. Precentral Gyrus (M1) & 4 & 54 & -14 & 44 & 9.26 & 0.012 \\
\hline \multirow{2}{*}{ R. Temporal Pole } & 1 & 4 & -24 & 48 & 9.02 & 0.017 \\
& 2 & 52 & 20 & -22 & 8.83 & 0.022 \\
\hline
\end{tabular}




\section{Discussion}

We used data from a virtual reality-based hand-target phase matching task to identify the hemodynamic and oscillatory correlates of performance (i.e., phase matching accuracy) monitoring under instructed task relevance of visual or proprioceptive hand position feedback. Our main result was a general, modality-independent response of the bilateral FO to poor phase matching accuracy, as evident from increased BOLD signal levels and increased gamma power. Furthermore, connectivity of the bilateral FO to the right PPC/IPL increased while participants executed the phase matching task with the visible virtual hand, compared to when they executed it with the real, unseen hand.

The observed general BOLD signal increase in the bilateral FO with task (phase matching) inaccuracy confirms observations of numerous previous studies, where BOLD signal in the FO increased in response to performance errors. For instance, the FO was activated by error trials vs correct trials in the Simon task (Danielmeier et al., 2011; Ham et al., 2013); in an antisaccade task (Klein et al., 2007); and in a flanker task (Eichele et al., 2008). Similarly, FO error-related BOLD signal increases were observed in visuomotor adaptation tasks (Grafton et al., 2008) and in response to tactile 'oddball' stimuli (Allen et al., 2016). Some studies found activation of the FO correlated positively with task performance (Bunge et al., 2015; Wager et al., 2005). This could, however, be explained with an general underlying function of FO activation in performance monitoring; acting not as an error signal per se, but as part of a mechanism to improve performance in response to errors (cf. Eichele et al., 2008). Thus, it has been proposed that neuronal activity in the FO may indicate the need for increased allocation of attentional resources to specific stimuli to achieve task-appropriate behaviour (Cieslik et al., 2015; Ham et al., 2013; Langner \& Eickhoff, 2013; Uddin, 2021). Additionally, due to FO's reciprocal connections to multiple sensory, limbic, and association areas (Sridharan et al., 2008), it may act as crucial 'relay' station for switching between different task relevant networks, e.g. switching from default network to executive control network (Klein et al., 2007; Menon \& Uddin, 2010; Sridharan et al., 2008; Ullsperger et al., 2010). 
The spectral correlates of task inaccuracy were expressed in the gamma frequency range; and, notably

in agreement with the BOLD signal increases, they were source-localized to the bilateral FO (as part of

larger sources in the IFG). A general correspondence and spatial co-localization of the BOLD signal and FO may exert causal influence over fronto-parietal areas within the gamma band.

In sum, and in light of the above literature, our fMRI and MEG results suggest that the FO is involved in performance monitoring during goal-directed hand movements. Notably, while most of the above studies used trial-by-trial designs, our study featured continuous movements; thus, our results complement previous literature in showing that the FO shows similar responses in task settings requiring 'on-line' performance monitoring and adjustment during manual actions. Specifically, we propose that FO activation (expressed through BOLD signal and gamma power increase) may have indicated a reaction to task inaccuracy or error, and a corresponding need for behavioural adjustment. significance, see Results). It is well established that posterior alpha inversely correlates with attention

333 and task engagement (Bacigalupo \& Luck, 2019; Sauseng et al., 2005; Thut, 2006; Yamagishi et al., 2003). 
In addition to increased activation of the bilateral FO, our fMRI connectivity analysis revealed that these areas also increased their functional coupling with the right PPC (peak located in the IPL) during the $\mathrm{VH}$ task (phase matching with vision) compared with the $\mathrm{RH}$ task (phase matching with proprioception). An fMRI study by Higo et al. (2011) had used a task requiring attention to faces, houses, or body parts; and found that the FO increased its functional coupling with visual areas processing the respective task relevant stimulus category. In our case, however, the FO's connectivity increase was not with primary and secondary visual cortices, which had shown task (attentional set)

(Andersen \& Buneo, 2002; Malhotra et al., 2009; Wolpert et al., 1998).

We propose that this result is related to the fact that visual hand movements were task relevant in the VH task, but had to be ignored in the $\mathrm{RH}$ task (where phase matching was done with proprioception). Thus, visual feedback was essential for correcting phase matching error in the VH task, but irrelevant in the RH task. Notably, FO connectivity was not significantly different during periods of visuoproprioceptive incongruence; neither did we find significant differences between congruent and incongruent conditions in the main fMRI GLM analysis. This suggests that the observed VH $>$ RH task dependent connectivity difference was related to the task-relevant modality being vision > proprioception per se, rather than to (in)congruence between vision and proprioception. This interpretation fits with previous work showing that the right PPC, specifically areas in the right IPL, are critical hubs for executing and correcting visually guided arm movements (Culham et al., 2003; Culham \& Valyear, 2006; Desmurget et al., 1999; Lane et al., 2011; Ogawa et al., 2007; Wenderoth et al., 2004). Potentially, this effect might have been enhanced by intrinsic differences between the modalities in relation to error detection; i.e., it might have been easier for participants to notice a phase matching error when focusing on visual action feedback ( $\mathrm{VH}$ task) than when focusing on proprioception ( $\mathrm{RH}$ 
proprioceptive ones (cf. van Beers et al., 1999); and because visual body position was easier to compare to the visually presented target (however, the target quantity was not visuo-spatial but abstract; i.e., the oscillatory growing-and-shrinking phase of the fixation dot). When we lowered the statistical threshold of the main GLM analysis to $p<0.005$, uncorrected, the bilateral FO (the PPI seed regions) and the right PPC (the PPI target region) showed a stronger correlation with task inaccuracy under the $\mathrm{VH}$ task compared with the RH task. Although this was a weak effect, it could mean that overall, errors were more easily processed (in those areas) in the VH task. Interestingly, participants were overall worse in the $\mathrm{RH}$ than in the $\mathrm{VH}$ task, and performance varied more strongly in the $\mathrm{RH}$ task; this could support the interpretation that proprioceptive performance monitoring was less efficient than when vision was used. This may also fit with previous reports of increased BOLD signal in the FO for error trials of which participants were aware, than unaware errors (Harsay et al., 2018; Klein et al., 2007). Specifically, Harsay et al. (2018) also observed increased functional connectivity of the FO to the PPC (bilaterally, in addition to the bilateral S1) during aware > unaware errors. In sum, we suggest that the increased connectivity between the $\mathrm{FO}$ and the right PPC during the $\mathrm{VH}>\mathrm{RH}$ task indicates the FO signalling an increased need for control and attentional and/or behavioural adjustment (following poor performance) to visuomotor regions in the right PPC; which could also be related to how easily those performance deficits could be detected.

The above speculations could also explain why we did not observe any connectivity increases of the FO during the RH > VH task. Accordingly, this could be because participants were less aware of their phase matching (in)accuracy when performing the task with the unseen real hand. Future work should evaluate this possibility with specific task designs.

Besides activations in the bilateral FO, we also found BOLD signal increases to poor phase matching in the SMA and the dIPFC (at the junction of middle frontal gyrus and frontal pole). Both areas have been strongly implied in performance monitoring in other contexts (Ullsperger et al., 2014). The SMA has been shown to respond to unexpected stimuli, e.g. surprising action outcomes (Krakauer et al., 2004; Sakai et al., 1999; Scangos et al., 2013; Ullsperger et al., 2010). BOLD signal in the SMA has previously 
been reported to correlate with positional error in a visuomotor learning task (Grafton et al., 2008) and in a continuous hand-target tracking (Limanowski et al., 2017). In line with the interpretation provided in these studies, the SMA activation we observed may indicate an updating of movement plans in response to poor detected phase matching. Similarly, the lateral PFC is considered a crucial part of sensorimotor hierarchy (Benchenane et al., 2011; Sokhadze et al., 2012), and is thought to contribute to performance monitoring and error detection; e.g., by preparing attentional task sets and comparing behavioural output against them (Cieslik et al., 2015; Danielmeier et al., 2011; Smith et al., 2019; Ullsperger \& von Cramon, 2004). In our experiment, the dIPFC activation could imply similar underlying 'high level' functions.

Conversely, we observed that BOLD signal in the contralateral M1 correlated positively with task accuracy. This effect could be related to the fact that higher task accuracy coincided with more pronounced hand movements; however, we had included movement amplitude as a regressor of no interest in our first-level GLMs, which should have largely accounted for this potential bias. Alternatively, this observation also aligns with the M1's known role in motor learning (Hardwick et al., 2013; Panico et al., 2021; Spampinato \& Celnik, 2017); with previous findings that M1 activity correlated with visuomotor adaptation performance (Della-Maggiore \& McIntosh, 2005) or with visuomotor target tracking performance (Ogawa et al., 2006); and with the fact that a perturbance of the M1 via transcranial magnetic stimulation (TMS) resulted in reduced sensorimotor adaption (Orban de Xivry et al., 2011).

Finally, it should be noted that our results should be compared to previous studies with some caution, since our task was designed around continuous movements; therefore, we could not isolate specific time points-and neuronal correlates - that would clearly correspond to specific cognitive or motor processes like e.g. error detection or correction. Future trial-by-trial task designs should therefore try to validate our interpretation. 
411 In conclusion, our results suggest a critical role for the bilateral FO in performance monitoring during manual action; and that following errors in visually guided manual action specifically, the FO may signal an increased need for control to visuomotor regions in the right PPC.

\section{Conflict of interest}

The authors declare no conflict of interest.

\section{Acknowledgments}

Funded by the German Research Foundation (DFG, Deutsche Forschungsgemeinschaft) as part of Germany's Excellence Strategy - EXC 2050/1 - Project ID 390696704 - Cluster of Excellence “Centre for Tactile Internet with Human-in-the-Loop" (CeTI) of Technische Universität Dresden. JL is funded by a Freigeist Fellowship of the VolkswagenStiftung (AZ 97-932). Figure 1 reprinted from: Limanowski et al., Neuroimage, 222, p. 117267, Copyright Elsevier (2020) under the terms of the Creative Commons CC-BY license. 


\section{References}

Allen, M., Fardo, F., Dietz, M. J., Hillebrandt, H., Friston, K. J., Rees, G., \& Roepstorff, A. (2016). Anterior insula coordinates hierarchical processing of tactile mismatch responses. Neurolmage, 127, 34-43. https://doi.org/10.1016/j.neuroimage.2015.11.030

Andersen, R. A., \& Buneo, C. A. (2002). Intentional Maps in Posterior Parietal Cortex. Annual Review of Neuroscience, 25(1), 189-220. https://doi.org/10.1146/annurev.neuro.25.112701.142922

Bacigalupo, F., \& Luck, S. J. (2019). Lateralized Suppression of Alpha-Band EEG Activity As a Mechanism of Target Processing. The Journal of Neuroscience, 39(5), 900-917. https://doi.org/10.1523/JNEUROSCI.0183-18.2018

Bastin, J., Deman, P., David, O., Gueguen, M., Benis, D., Minotti, L., Hoffman, D., Combrisson, E., Kujala, J., Perrone-Bertolotti, M., Kahane, P., Lachaux, J.-P., \& Jerbi, K. (2016). Direct Recordings from Human Anterior Insula Reveal its Leading Role within the Error-Monitoring Network. Cerebral Cortex, bhv352. https://doi.org/10.1093/cercor/bhv352

Belardinelli, P., Ortiz, E., Barnes, G., Noppeney, U., \& Preissl, H. (2012). Source Reconstruction Accuracy of MEG and EEG Bayesian Inversion Approaches. PLoS ONE, 7(12), e51985. https://doi.org/10.1371/journal.pone.0051985

Benchenane, K., Tiesinga, P. H., \& Battaglia, F. P. (2011). Oscillations in the prefrontal cortex: A gateway to memory and attention. Current Opinion in Neurobiology, 21(3), 475-485. https://doi.org/10.1016/j.conb.2011.01.004

Billeke, P., Ossandon, T., Perrone-Bertolotti, M., Kahane, P., Bastin, J., Jerbi, K., Lachaux, J.-P., \& Fuentealba, P. (2020). Human Anterior Insula Encodes Performance Feedback and Relays Prediction Error to the Medial Prefrontal Cortex. Cerebral Cortex, 30(7), 4011-4025. https://doi.org/10.1093/cercor/bhaa017

Brovelli, A., Lachaux, J.-P., Kahane, P., \& Boussaoud, D. (2005). High gamma frequency oscillatory activity dissociates attention from intention in the human premotor cortex. Neurolmage, 28(1), 154-164. https://doi.org/10.1016/j.neuroimage.2005.05.045 
Bunge, S. A., Dudukovic, N. M., Thomason, M. E., Vaidya, C. J., \& Gabrieli, J. D. E. (2015). Immature Frontal Lobe Contributions to Cognitive Control in Children: Evidence from fMRI. 25.

Castelhano, J., Duarte, I. C., Wibral, M., Rodriguez, E., \& Castelo-Branco, M. (2014). The dual facet of gamma oscillations: Separate visual and decision making circuits as revealed by simultaneous EEG/fMRI. Human Brain Mapping, 35(10), 5219-5235. https://doi.org/10.1002/hbm.22545

Chand, G. B., \& Dhamala, M. (2017). Interactions between the anterior cingulate-insula network and the fronto-parietal network during perceptual decision-making. Neurolmage, 152, 381-389. https://doi.org/10.1016/j.neuroimage.2017.03.014

Cieslik, E. C., Mueller, V. I., Eickhoff, C. R., Langner, R., \& Eickhoff, S. B. (2015). Three key regions for supervisory attentional control: Evidence from neuroimaging meta-analyses. Neuroscience \& Biobehavioral Reviews, 48, 22-34. https://doi.org/10.1016/j.neubiorev.2014.11.003

Clayton, M. S., Yeung, N., \& Cohen Kadosh, R. (2015). The roles of cortical oscillations in sustained attention. Trends in Cognitive Sciences, 19(4), 188-195. https://doi.org/10.1016/j.tics.2015.02.004

Culham, J. C., Danckert, S. L., Souza, J. F. X. D., Gati, J. S., Menon, R. S., \& Goodale, M. A. (2003). Visually guided grasping produces fMRI activation in dorsal but not ventral stream brain areas. Experimental Brain Research, 153(2), 180-189. https://doi.org/10.1007/s00221-003$1591-5$

Culham, J. C., \& Valyear, K. F. (2006). Human parietal cortex in action. Current Opinion in Neurobiology, 16(2), 205-212. https://doi.org/10.1016/j.conb.2006.03.005

Danielmeier, C., Eichele, T., Forstmann, B. U., Tittgemeyer, M., \& Ullsperger, M. (2011). Posterior Medial Frontal Cortex Activity Predicts Post-Error Adaptations in Task-Related Visual and Motor Areas. Journal of Neuroscience, 31(5), 1780-1789. https://doi.org/10.1523/JNEUROSCI.4299-10.2011 
Della-Maggiore, V., \& Mclntosh, A. R. (2005). Time Course of Changes in Brain Activity and Functional Connectivity Associated With Long-Term Adaptation to a Rotational Transformation. Journal of Neurophysiology, 93(4), 2254-2262. https://doi.org/10.1152/jn.00984.2004

Desmurget, M., Epstein, C. M., Turner, R. S., Prablanc, C., Alexander, G. E., \& Grafton, S. T. (1999). Role of the posterior parietal cortex in updating reaching movements to a visual target. Nature Neuroscience, 2(6), 563-567. https://doi.org/10.1038/9219

Diedrichsen, J. (2005). Neural Correlates of Reach Errors. Journal of Neuroscience, 25(43), 99199931. https://doi.org/10.1523/JNEUROSCI.1874-05.2005

Donner, T. H., \& Siegel, M. (2011). A framework for local cortical oscillation patterns. Trends in Cognitive Sciences, 15(5), 191-199. https://doi.org/10.1016/j.tics.2011.03.007

Eichele, T., Debener, S., Calhoun, V. D., Specht, K., Engel, A. K., Hugdahl, K., von Cramon, D. Y., \& Ullsperger, M. (2008). Prediction of human errors by maladaptive changes in event-related brain networks. Proceedings of the National Academy of Sciences, 105(16), 6173-6178. https://doi.org/10.1073/pnas.0708965105

Eickhoff, S. B., Stephan, K. E., Mohlberg, H., Grefkes, C., Fink, G. R., Amunts, K., \& Zilles, K. (2005). A new SPM toolbox for combining probabilistic cytoarchitectonic maps and functional imaging data. Neurolmage, 25(4), 1325-1335. https://doi.org/10.1016/j.neuroimage.2004.12.034

Foucher, J. R., Otzenberger, H., \& Gounot, D. (2003). The BOLD response and the gamma oscillations respond differently than evoked potentials: An interleaved EEG-fMRI study. BMC Neuroscience, 11.

Friston, K. J., Buechel, C., Fink, G. R., Morris, J., Rolls, E., \& Dolan, R. J. (1997). Psychophysiological and Modulatory Interactions in Neuroimaging. Neurolmage, 6(3), 218-229. https://doi.org/10.1006/nimg.1997.0291

Friston, K. J., Preller, K. H., Mathys, C., Cagnan, H., Heinzle, J., Razi, A., \& Zeidman, P. (2019). Dynamic causal modelling revisited. Neurolmage, 199, 730-744. https://doi.org/10.1016/j.neuroimage.2017.02.045 
Grafton, S. T., Schmitt, P., Van Horn, J., \& Diedrichsen, J. (2008). Neural substrates of visuomotor learning based on improved feedback control and prediction. Neurolmage, 39(3), 13831395. https://doi.org/10.1016/j.neuroimage.2007.09.062

Grent-'t-Jong, T., Oostenveld, R., Jensen, O., Medendorp, W. P., \& Praamstra, P. (2013). Oscillatory dynamics of response competition in human sensorimotor cortex. Neurolmage, 83, 27-34. https://doi.org/10.1016/j.neuroimage.2013.06.051

Ham, T., Leff, A., de Boissezon, X., Joffe, A., \& Sharp, D. J. (2013). Cognitive Control and the Salience Network: An Investigation of Error Processing and Effective Connectivity. Journal of Neuroscience, 33(16), 7091-7098. https://doi.org/10.1523/JNEUROSCI.4692-12.2013

Hardwick, R. M., Rottschy, C., Miall, R. C., \& Eickhoff, S. B. (2013). A quantitative meta-analysis and review of motor learning in the human brain. Neurolmage, 67, 283-297. https://doi.org/10.1016/j.neuroimage.2012.11.020

Harsay, H. A., Cohen, M. X., Spaan, M., Weeda, W. D., Nieuwenhuis, S., \& Ridderinkhof, K. R. (2018). Error blindness and motivational significance: Shifts in networks centering on anterior insula co-vary with error awareness and pupil dilation. Behavioural Brain Research, 355, 24-35. https://doi.org/10.1016/j.bbr.2017.10.030

Henson, R. N., Flandin, G., Friston, K. J., \& Mattout, J. (2010). A Parametric Empirical Bayesian framework for fMRI-constrained MEG/EEG source reconstruction. Human Brain Mapping, 31(10), 1512-1531. https://doi.org/10.1002/hbm.20956

Higo, T., Mars, R. B., Boorman, E. D., Buch, E. R., \& Rushworth, M. F. S. (2011). Distributed and causal influence of frontal operculum in task control. Proceedings of the National Academy of Sciences, 108(10), 4230-4235. https://doi.org/10.1073/pnas.1013361108

Hipp, J. F., Hawellek, D. J., Corbetta, M., Siegel, M., \& Engel, A. K. (2012). Large-scale cortical correlation structure of spontaneous oscillatory activity. Nature Neuroscience, 15(6), 884890. https://doi.org/10.1038/nn.3101 
Jensen, O., Kaiser, J., \& Lachaux, J.-P. (2007). Human gamma-frequency oscillations associated with attention and memory. Trends in Neurosciences, 30(7), 317-324. https://doi.org/10.1016/j.tins.2007.05.001

Kilner, J. M., \& Friston, K. J. (2010). Topological inference for EEG and MEG. The Annals of Applied Statistics, 4(3). https://doi.org/10.1214/10-AOAS337

Klein, T. A., Endrass, T., Kathmann, N., Neumann, J., von Cramon, D. Y., \& Ullsperger, M. (2007). Neural correlates of error awareness. Neurolmage, 34(4), 1774-1781. https://doi.org/10.1016/j.neuroimage.2006.11.014

Klein, T. A., Ullsperger, M., \& Danielmeier, C. (2013). Error awareness and the insula: Links to neurological and psychiatric diseases. Frontiers in Human Neuroscience, 7. https://doi.org/10.3389/fnhum.2013.00014

Krakauer, J. W., Ghilardi, M.-F., Mentis, M., Barnes, A., Veytsman, M., Eidelberg, D., \& Ghez, C. (2004). Differential Cortical and Subcortical Activations in Learning Rotations and Gains for Reaching: A PET Study. Journal of Neurophysiology, 91(2), 924-933. https://doi.org/10.1152/jn.00675.2003

Lane, A. R., Smith, D. T., Schenk, T., \& Ellison, A. (2011). The Involvement of Posterior Parietal Cortex in Feature and Conjunction Visuomotor Search. Journal of Cognitive Neuroscience, 23(8), 1964-1972. https://doi.org/10.1162/jocn.2010.21576

Langner, R., \& Eickhoff, S. B. (2013). Sustaining attention to simple tasks: A meta-analytic review of the neural mechanisms of vigilant attention. Psychological Bulletin, 139(4), 870-900. https://doi.org/10.1037/a0030694

Lebar, N., Danna, J., Moré, S., Mouchnino, L., \& Blouin, J. (2017). On the neural basis of sensory weighting: Alpha, beta and gamma modulations during complex movements. Neurolmage, 150, 200-212. https://doi.org/10.1016/j.neuroimage.2017.02.043

Limanowski, J., \& Friston, K. (2020). Attentional Modulation of Vision Versus Proprioception During Action. Cerebral Cortex, 30(3), 1637-1648. https://doi.org/10.1093/cercor/bhz192 
Limanowski, J., Kirilina, E., \& Blankenburg, F. (2017). Neuronal correlates of continuous manual tracking under varying visual movement feedback in a virtual reality environment. Neurolmage, 146, 81-89. https://doi.org/10.1016/j.neuroimage.2016.11.009

Limanowski, J., Litvak, V., \& Friston, K. (2020). Cortical beta oscillations reflect the contextual gating of visual action feedback. Neurolmage, 222, 117267. https://doi.org/10.1016/j.neuroimage.2020.117267

Litvak, V., \& Friston, K. (2008). Electromagnetic source reconstruction for group studies. Neurolmage, 42(4), 1490-1498. https://doi.org/10.1016/j.neuroimage.2008.06.022

López, J. D., Litvak, V., Espinosa, J. J., Friston, K., \& Barnes, G. R. (2014). Algorithmic procedures for Bayesian MEG/EEG source reconstruction in SPM. Neurolmage, 84, 476-487. https://doi.org/10.1016/j.neuroimage.2013.09.002

Malhotra, P., Coulthard, E. J., \& Husain, M. (2009). Role of right posterior parietal cortex in maintaining attention to spatial locations over time. Brain, 132(3), 645-660. https://doi.org/10.1093/brain/awn350

Menon, V., \& Uddin, L. Q. (2010). Saliency, switching, attention and control: A network model of insula function. Brain Structure and Function, 214(5-6), 655-667. https://doi.org/10.1007/s00429-010-0262-0

Moran, R. J., Stephan, K. E., Kiebel, S. J., Rombach, N., O’Connor, W. T., Murphy, K. J., Reilly, R. B., \& Friston, K. J. (2008). Bayesian estimation of synaptic physiology from the spectral responses of neural masses. 13.

Ogawa, K., Inui, T., \& Sugio, T. (2006). Separating brain regions involved in internally guided and visual feedback control of moving effectors: An event-related fMRI study. Neurolmage, 32(4), 1760-1770. https://doi.org/10.1016/j.neuroimage.2006.05.012

Ogawa, K., Inui, T., \& Sugio, T. (2007). Neural Correlates of State Estimation in Visually Guided Movements: An Event-Related FMRI Study. Cortex, 43(3), 289-300. https://doi.org/10.1016/S0010-9452(08)70455-6 
Orban de Xivry, J.-J., Criscimagna-Hemminger, S. E., \& Shadmehr, R. (2011). Contributions of the Motor Cortex to Adaptive Control of Reaching Depend on the Perturbation Schedule. Cerebral Cortex, 21(7), 1475-1484. https://doi.org/10.1093/cercor/bhq192

O'Reilly, J. X., Woolrich, M. W., Behrens, T. E. J., Smith, S. M., \& Johansen-Berg, H. (2012). Tools of the trade: Psychophysiological interactions and functional connectivity. Social Cognitive and Affective Neuroscience, 7(5), 604-609. https://doi.org/10.1093/scan/nss055

Panico, F., Fleury, L., Trojano, L., \& Rossetti, Y. (2021). Prism Adaptation in M1. Journal of Cognitive Neuroscience, 33(4), 563-573. https://doi.org/10.1162/jocn_a_01668

Sakai, K., Hikosaka, O., Miyauchi, S., Sasaki, Y., Fujimaki, N., \& Pütz, B. (1999). Presupplementary Motor Area Activation during Sequence Learning Reflects Visuo-Motor Association. The Journal of Neuroscience, 19(10), RC1-RC1. https://doi.org/10.1523/JNEUROSCI.19-10j0002.1999

Sauseng, P., Klimesch, W., Stadler, W., Schabus, M., Doppelmayr, M., Hanslmayr, S., Gruber, W. R., \& Birbaumer, N. (2005). A shift of visual spatial attention is selectively associated with human EEG alpha activity. European Journal of Neuroscience, 22(11), 2917-2926. https://doi.org/10.1111/j.1460-9568.2005.04482.x

Scangos, K. W., Aronberg, R., \& Stuphorn, V. (2013). Performance monitoring by presupplementary and supplementary motor area during an arm movement countermanding task. Journal of Neurophysiology, 109(7), 1928-1939. https://doi.org/10.1152/jn.00688.2012

Seeley, W. W., Menon, V., Schatzberg, A. F., Keller, J., Glover, G. H., Kenna, H., Reiss, A. L., \& Greicius, M. D. (2007). Dissociable Intrinsic Connectivity Networks for Salience Processing and Executive Control. Journal of Neuroscience, 27(9), 2349-2356. https://doi.org/10.1523/JNEUROSCI.5587-06.2007

Siegel, M., Donner, T. H., \& Engel, A. K. (2012). Spectral fingerprints of large-scale neuronal interactions. Nature Reviews Neuroscience, 13(2), 121-134. https://doi.org/10.1038/nrn3137 
Smith, E. H., Horga, G., Yates, M. J., Mikell, C. B., Banks, G. P., Pathak, Y. J., Schevon, C. A., McKhann, G. M., Hayden, B. Y., Botvinick, M. M., \& Sheth, S. A. (2019). Widespread temporal coding of cognitive control in the human prefrontal cortex. Nature Neuroscience, 22(11), 1883-1891. https://doi.org/10.1038/s41593-019-0494-0

Sober, S. J., \& Sabes, P. N. (2005). Flexible strategies for sensory integration during motor planning. Nature Neuroscience, 8(4), 490-497. https://doi.org/10.1038/nn1427

Sokhadze, E. M., Baruth, J. M., Sears, L., Sokhadze, G. E., El-Baz, A. S., \& Casanova, M. F. (2012). Prefrontal Neuromodulation Using rTMS Improves Error Monitoring and Correction Function in Autism. Applied Psychophysiology and Biofeedback, 37(2), 91-102. https://doi.org/10.1007/s10484-012-9182-5

Spampinato, D., \& Celnik, P. (2017). Temporal dynamics of cerebellar and motor cortex physiological processes during motor skill learning. Scientific Reports, 7(1), 40715. https://doi.org/10.1038/srep40715

Sridharan, D., Levitin, D. J., \& Menon, V. (2008). A critical role for the right fronto-insular cortex in switching between central-executive and default-mode networks. Proceedings of the National Academy of Sciences, 105(34), 12569-12574. https://doi.org/10.1073/pnas.0800005105

Suminski, A. J., Rao, S. M., Mosier, K. M., \& Scheidt, R. A. (2007). Neural and Electromyographic Correlates of Wrist Posture Control. Journal of Neurophysiology, 97(2), 1527-1545. https://doi.org/10.1152/jn.01160.2006

Thomson, D. J. (1982). Spectrum estimation and harmonic analysis. Proceedings of the IEEE, 7O(9), 1055-1096. https://doi.org/10.1109/PROC.1982.12433

Thut, G. (2006). -Band Electroencephalographic Activity over Occipital Cortex Indexes Visuospatial Attention Bias and Predicts Visual Target Detection. Journal of Neuroscience, 26(37), 94949502. https://doi.org/10.1523/JNEUROSCI.0875-06.2006 
Uddin, L. Q. (2021). Cognitive and behavioural flexibility: Neural mechanisms and clinical considerations. Nature Reviews Neuroscience, 22(3), 167-179. https://doi.org/10.1038/s41583-021-00428-w

Ullsperger, M., Danielmeier, C., \& Jocham, G. (2014). Neurophysiology of Performance Monitoring and Adaptive Behavior. Physiological Reviews, 94(1), 35-79. https://doi.org/10.1152/physrev.00041.2012

Ullsperger, M., Harsay, H. A., Wessel, J. R., \& Ridderinkhof, K. R. (2010). Conscious perception of errors and its relation to the anterior insula. Brain Structure and Function, 214(5-6), 629643. https://doi.org/10.1007/s00429-010-0261-1

Ullsperger, M., \& von Cramon, D. Y. (2004). Neuroimaging of Performance Monitoring: Error Detection and Beyond. Cortex, 40(4-5), 593-604. https://doi.org/10.1016/S00109452(08)70155-2

van Beers, R. J., Sittig, A. C., \& Gon, J. J. D. van der. (1999). Integration of Proprioceptive and Visual Position-Information: An Experimentally Supported Model. Journal of Neurophysiology, 81(3), 1355-1364. https://doi.org/10.1152/jn.1999.81.3.1355

Wager, T. D., Sylvester, C.-Y. C., Lacey, S. C., Nee, D. E., Franklin, M., \& Jonides, J. (2005). Common and unique components of response inhibition revealed by fMRI. Neurolmage, 27(2), 323340. https://doi.org/10.1016/j.neuroimage.2005.01.054

Wenderoth, N., Debaere, F., Sunaert, S., Hecke, P. van, \& Swinnen, S. P. (2004). Parieto-premotor Areas Mediate Directional Interference During Bimanual Movements. Cerebral Cortex, 14(10), 1153-1163. https://doi.org/10.1093/cercor/bhh075

Wolpert, D. M., Goodbody, S. J., \& Husain, M. (1998). Maintaining internal representations: The role of the human superior parietal lobe. Nature Neuroscience, 1(6), 529-533. https://doi.org/10.1038/2245 
bioRxiv preprint doi: https://doi.org/10.1101/2021.10.21.465314; this version posted October 22, 2021. The copyright holder for this preprint (which was not certified by peer review) is the author/funder, who has granted bioRxiv a license to display the preprint in perpetuity. It is made available under aCC-BY-NC-ND 4.0 International license. 